\title{
Reactivation-dependent amnesia in Pavlovian approach and instrumental transfer
}

\author{
Jonathan L.C. Lee ${ }^{1,2}$ and Barry J. Everitt \\ Behavioural and Clinical Neuroscience Institute, Department of Experimental Psychology, University of Cambridge, \\ Cambridge CB2 3EB, United Kingdom
}

\begin{abstract}
The theory of memory reconsolidation relates to the hypothesized restabilisation process that occurs following the reactivation of a memory through retrieval. Thus the demonstration of reactivation-dependent amnesia for a previously acquired memory is a prerequisite for showing that such a memory undergoes reconsolidation. Here we show that the appetitive Pavlovian representations that underlie Pavlovian approach and Pavlovian-instrumental transfer are destabilized following their retrieval. This reactivation-dependent amnesia demonstrates that the general motivational or incentive properties of appetitive conditioned stimuli, as well as their conditioned reinforcing properties, can be reduced by blocking memory reconsolidation.
\end{abstract}

Memories are not only vulnerable to disruption following their acquisition, but also during a limited time after their retrieval or reactivation. Thus old memories can be disrupted by administering amnestic agents at the time of re-exposure to salient trainingrelated cues (e.g., Nader et al. 2000). Such cue-dependent or reactivation-dependent amnesia is thought to reflect a disruption of a memory reconsolidation process that restabilises memories following their reactivation (Lewis 1979; Nader 2003). While much of the research into memory reconsolidation has used rapidly acquired aversive memory paradigms, there is an emerging literature concerning the reconsolidation of appetitive Pavlovian memories.

In rodents, several studies have investigated the reconsolidation of addictive drug-associated Pavlovian memories with a view to potential treatments for drug addiction (Lee et al. 2005, 2006a; Miller and Marshall 2005; Milekic et al. 2006; Valjent et al. 2006; Milton et al. 2008). An appetitive conditioned stimulus (CS) acquires both sensory-specific properties and more general incentive or motivational attributes when associated with an unconditioned stimulus such as food (Konorski 1967; Everitt et al. 2000). While we have tested specifically the reconsolidation of the sensory-specific conditioned reinforcing properties of drugassociated stimuli (Lee et al. 2005; Milton et al. 2008), many studies have used a conditioned place preference procedure (Miller and Marshall 2005; Milekic et al. 2006; Valjent et al. 2006) that is more difficult to characterize in terms of its psychological basis (Everitt et al. 2000). A place preference might be mediated by conditioned reinforcement by the drug-associated context or cues, or by a simple Pavlovian approach to those stimuli. It therefore remains unclear whether the memories mediating the general motivational properties of appetitive conditioned stimuli undergo reconsolidation following their retrieval.

The general motivational properties of CSs can be assessed in two ways: general Pavlovian-instrumental transfer (PIT) and Pavlovian approach or "sign-tracking" as measured in autoshaping procedures (Everitt et al. 2000; Cardinal et al. 2002). We have shown previously that these tasks depend upon different neural substrates from those that mediate conditioned reinforcement (Cador et al. 1989; Robledo et al. 1996; Parkinson et al. 2000a;

\footnotetext{
'Present address: University of Birmingham, School of Psychology, Edgbaston, Birmingham B152TT, UK.

${ }^{2}$ Corresponding author.

E-mail joff@cantab.net; fax +44-121-414-4897.

Article is online at http://www.learnmem.org/cgi/doi/10.1101/lm.1029808.
}

Hall et al. 2001). While Blaiss and Janak (2007) have reported that post-reactivation protein synthesis inhibition did not impair Pavlovian conditioned approach, this study used a goal-tracking procedure that may not test specifically the general motivational properties of an appetitive CS. We have shown that systemic administration of the NMDA receptor antagonist MK-801 (Lee and Everitt 2008) or the $\beta$-adrenergic receptor antagonist propranolol (Milton et al. 2008) impairs the reconsolidation of CSsucrose memories as assessed in a procedure that isolates the conditioned reinforcing properties of the CS. Therefore, in the present study we have used MK-801 and propranolol to investigate reactivation-dependent amnesia in Pavlovian-instrumental transfer and autoshaping procedures, and thereby determine whether the memories mediating these general appetitive properties encoded by Pavlovian CSs undergo reconsolidation and depend upon glutamatergic and $\beta$-adrenergic mechanisms.

\section{Results}

\section{Experiment 1: Pavlovian-instrumental transfer}

There were no differences between the groups either during instrumental acquisition or Pavlovian training. All groups acquired the lever press response over the $9 \mathrm{~d}$ of training (Fig. 1A; Group $\times$ Session: $F_{(11,94)}=1.47, P>0.15$; Group: $\left.F<1\right)$ and demonstrated equivalent levels of Pavlovian approach during conditioning (Fig. 1B; Group $\times$ Session: $F_{(18,158)}=1.01, P>0.44$; Group: $\left.F_{(4,35)}=1.44, P>0.24\right)$. Moreover, ANOVA of performance during the memory reactivation session following drug treatment revealed no significant acute effects of MK-801 or propranolol upon Pavlovian approach (data not shown; $F<1$ ).

The administration of MK-801, but not propranolol, prior to CS reexposure resulted in a subsequent deficit in the ability of that CS to potentiate ongoing instrumental responding (Fig. 2). An overall three-way ANOVA (Fig. 2A,B) revealed that there was a significant Reactivation $\times$ MK- $801 \times \mathrm{CS}$ interaction $\left(F_{(1,28)}=7.59, P<0.02\right)$, but no MK-801 $\times$ CS interaction $\left(F_{(1,28)}=2.69, P>0.11\right)$. Neither was there an MK-801 $\times$ CS interaction when the nonreactivated condition was analyzed alone in a two-way ANOVA $\left(F_{(1,14)}=1.22, P>0.28\right)$. Therefore, whereas both saline and MK-801 treated rats responded on the lever at elevated levels in the presence of the CS+ compared to the neutral CS - in the nonreactivated condition (CS: $F_{(1,14)}=36.01$, $P<0.01)$, the combination of MK-801 injection and CS reexposure resulted in a lack of Pavlovian-instrumental transfer measured subsequently (CS: $F<1$ ). In contrast, saline treated, CS 
A

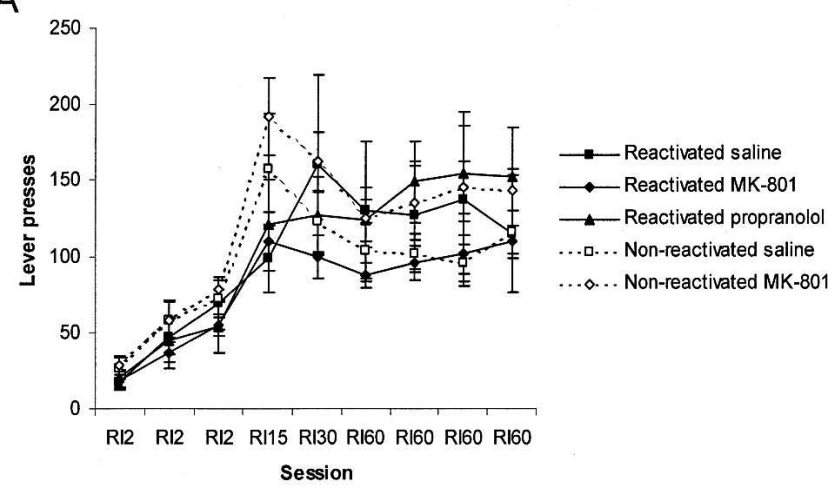

B

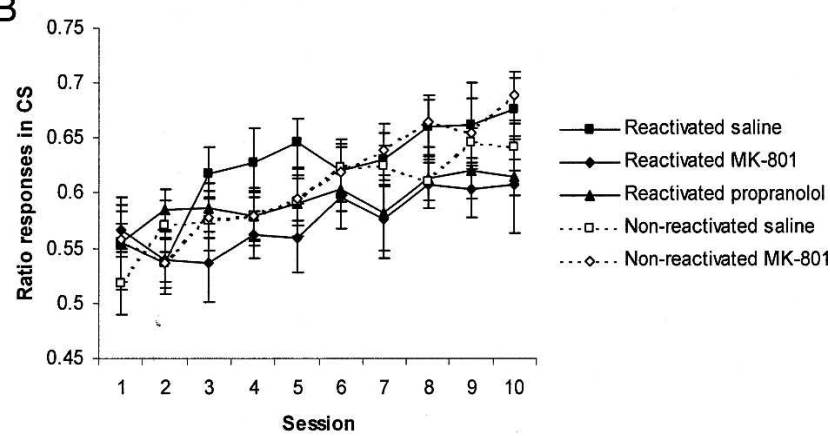

Figure 1. Acquisition of instrumental and Pavlovian conditioning prior to test for Pavlovian-instrumental transfer. Rats acquired over sessions the lever press response for liquid sucrose reward $(A)$ and subsequently developed a preferential approach to the reward location during the presentation of the CS $(B)$. There were no differences between the groups. Data presented as mean \pm SEM ( $n=7-9$ per group).

reexposed rats demonstrated a robust enhancement in the presence of the CS+ relative to the CS $-\left(F_{(1,7)}=12.81, P<0.01\right)$. This reactivation-dependent impairment following MK-801 treatment demonstrates that the Pavlovian representations that underlie PIT undergo reconsolidation following their retrieval in a manner dependent upon NMDA receptor-mediated signaling. Moreover, the failure of systemic propranolol to disrupt CS+ potentiated instrumental responding when injected prior to a memory reactivation session (two-way ANOVA; Propranolol $\times$ CS: $F_{(1,14)}=1.55, P>0.23$; Propranolol: $F<1$ ) shows that this same reconsolidation process is not critically dependent upon $\beta$-adrenergic receptor-mediated neurotransmission.

There was also a significant reactivation-dependent effect of MK-801 upon nosepoke responses during the PIT test (Fig. 3; Reactivation $\times$ MK-801 $\times$ CS: $F_{(1,28)}=13.14, P<0.01$; MK$801 \times$ CS: $F<1)$. However, this interaction was in fact driven by a higher level of nosepokes in rats treated with MK-801 compared to those injected with saline in the nonreactivated condition $\left(\right.$ MK-801 $\times$ CS: $\left.F_{(1,14)}=12.27, P<0.01\right)$, whereas the effect of MK-801 administration concurrent with memory reactivation did not reach significance $\left(\mathrm{MK}-801 \times \mathrm{CS}: F_{(1,14)}=3.77\right.$, $P=0.072$ ). Furthermore, propranolol treatment also had no effect on subsequent discriminated nosepoking (Propranolol $\times$ CS: $\left.F_{(1,14)}=1.94, P=0.18\right)$. While such a measure of discriminated approach has been used previously as indicative of Pavlovian approach (Parkinson et al. 1999), a more specific test involves the autoshaping procedure, in which "sign-tracking" or approach to the CS can be discriminated from "goal-tracking" or approach to the reward.

\section{Experiment 2: Autoshaping}

There were no differences between the groups during the acquisition of autoshaping (Fig. 4). The reactivated groups acquired discriminated approach to the CS+ over the 10 sessions of training $\left(\mathrm{CS}: F_{(1,18)}=38.15, P<0.01\right.$; CS $\times$ Session: $F_{(9,162)}=10.32$, $P<0.01$ ), with no differences between the groups (Group: $F<1$; $\mathrm{CS} \times$ Group: $F<1$; CS $\times$ Session $\times$ Group: $F<1$ ). Moreover, analysis of the acquisition data including the groups administered MK-801 in the absence of subsequent memory reactivation revealed no main effects or interactions involving the factors MK-801 and Reactivation (all Fs $<1$ ). Furthermore, ANOVA of performance during the memory reactivation session following drug treatment revealed no significant acute effects of MK-801 or propranolol (data not shown; Group: $F_{(2,18)}=3.38, P>0.05$; $\mathrm{CS} \times$ Group: $F<1)$.

The administration of MK-801, but not propranolol, prior to CS re-exposure also resulted in a subsequent deficit in the automatic discriminated Pavlovian approach to the CS+ (Fig. 5). An overall three-way ANOVA (Fig. 5A,B) revealed that there was a significant Reactivation $\times$ MK-801 $\times$ CS interaction $\left(F_{(1,22)}=10.46, P<0.01\right)$, as well as a significant MK-801 $\times \mathrm{CS}$ interaction $\left(F_{(1,22)}=5.15, P<0.04\right)$. However, there was no main effect of MK-801 $(F<1)$, and a two-way ANOVA of the nonreactivated groups alone showed that they did not differ from each other (MK-801 × CS: $F<1$; MK-801: $F<1$ ). Therefore, whereas both saline and MK-801 treated rats approached the CS+ more than the CS - in the nonreactivated condition (CS: $\left.F_{(1,14)}=36.01, P<0.01\right)$, the combination of MK-801 injection and CS reexposure resulted in a lack of discriminated approach to the CS+ measured subsequently (CS: $F<1$ ). In contrast, saline treated, CS reexposed rats demonstrated a clear preference for the CS+ over the CS $-\left(F_{(1,6)}=21.17, P<0.01\right)$. This reactivationdependent impairment following MK-801 treatment demonstrates that the Pavlovian representations that underlie autoshaping undergo reconsolidation following their retrieval in a manner dependent upon NMDA receptor-mediated signaling. Moreover, similar to PIT, the failure of systemic propranolol to disrupt autoshaping when injected prior to a memory reactivation session (two-way ANOVA; $F \mathrm{~s}<1$ ) shows that this reconsolidation process does not require $\beta$-adrenergic receptor-mediated neurotransmission.
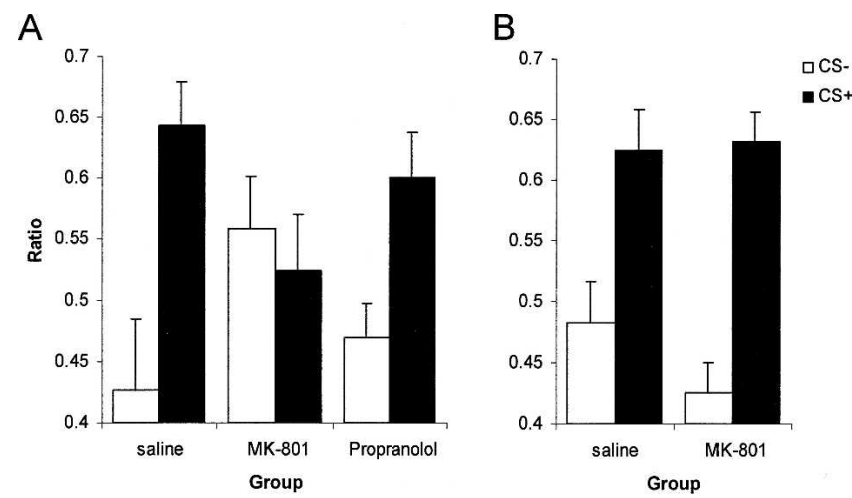

Figure 2. MK-801, but not propranolol, impaired subsequent Pavlovian-instrumental transfer. The ratio of instrumental lever press responding during the CS relative to the previous ISI period was compared for the $\mathrm{CS}+$ and the neutral $\mathrm{CS}-$. (A) While rats administered saline or propranolol prior to memory reactivation showed greater responding to the CS+ than the CS - , the administration of MK-801 eliminated such selective responding ( $n=8$ for each group). (B) The effects of MK- 801 were critically dependent upon memory reactivation, as omission of the reactivation session resulted in MK-801 having no effect on subsequent discriminative responding (MK-801: $n=7$; saline: $n=9$ ). Data presented as mean + SEM. 
A

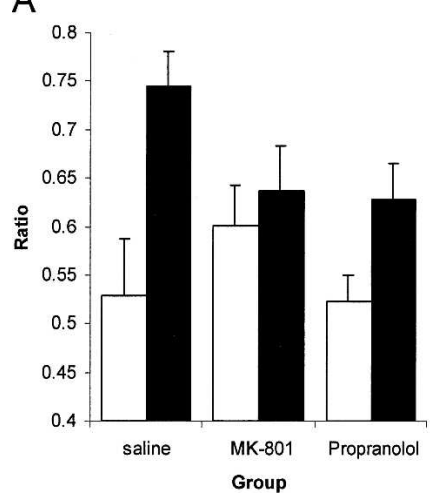

B

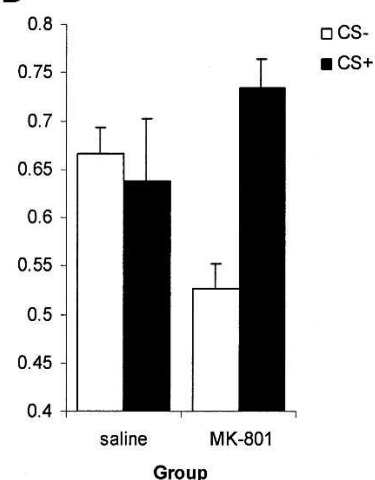

Figure 3. Nosepoke responding during the $C S+$ and $C S-$ in the Pavlovian-instrumental transfer test. MK-801, but not propranolol, administered prior to memory reactivation attenuated subsequent selective nosepoke approaches during the $\mathrm{CS}+(A ; n=8$ for each group). However, while rats treated with MK-801 in the absence of memory reactivation displayed a strong selective preference for the $\mathrm{CS}_{+}$, control rats administered with saline did not ( $B$; MK-801: $n=7$; saline: $n=9$ ). Data presented as mean + SEM.

\section{Discussion}

The results of these experiments demonstrate that general appetitive Pavlovian memory representations are disrupted following their acquisition in a memory reactivation-dependent manner. We used both a general Pavlovian-instrumental transfer and a sign-tracking, autoshaping procedure and showed that the reconsolidation of the memories underlying these behavioral responses were impaired by the systemic administration at memory reactivation of the NMDA receptor antagonist MK-801, but not the $\beta$-adrenergic receptor antagonist propranolol, at the doses tested. The reductions in performance indicate that the reconsolidation of the appetitive memory representations, rather than their extinction, was impaired. Thus while the reactivation sessions were long in absolute temporal terms, they were sufficiently brief relative to the extensive training to engage preferentially reconsolidation and not extinction mechanisms (Eisenberg et al. 2003).

The Pavlovian-instrumental transfer procedure used here measures the impact of Pavlovian conditioned stimuli upon instrumental performance (Hall et al. 2001). Previously conditioned appetitive Pavlovian stimuli enhance the rate of instrumental responding (Estes 1943; Lovibond 1983). Although in the present study the liquid sucrose reinforcer was common to both the Pavlovian CS and the instrumental response, the absence of a competing reinforcer with different associated CS and instrumental response renders the transfer effect studied here akin to the general transfer effect studied by Corbit and Balleine (2005), both of which depend upon processing in the central nucleus of the amygdala (Hall et al. 2001; Corbit and Balleine 2005). Therefore, the effect of MK-801 to impair Pavlovian instrumen-

tal transfer when administered systemically at the time of a prior memory reactivation session indicates that general appetitive Pavlovian memory representations undergo memory reconsolidation in a manner dependent upon glutamatergic signaling at NMDA receptors.

The pairing of a CS with a rewarding outcome results in the CS acquiring motivational properties that not only potentiate instrumental responding, but also elicit automatic, or Pavlovian, approach to the CS itself. Such autoshaping behavior is measured in the current procedure (adapted from Di Ciano et al. 2001) by discriminated approach to and contact with the compound lever/light CS+. Systemic administration of MK-801, but not propranolol, prior to a memory reactivation session entailing reexposure to both the paired CS+ and unpaired CS - abolished discriminated approach to the $\mathrm{CS}+$ in a subsequent probe test. Therefore, the memory representation mediating the motivational properties of an appetitive Pavlovian CS also undergoes memory reconsolidation in a NMDA receptor-dependent manner. This finding contrasts somewhat with the failure of postreactivation protein synthesis inhibition to impair memory reconsolidation in a Pavlovian conditioned approach procedure (Blaiss and Janak 2007). Importantly, the two procedures differ in the nature of the approach response. Blaiss and Janak (2007) measured goal-tracking behavior during CS presentation, rather than the sign-tracking CS approach tested here. While a measure of goal-tracking was embedded within our Pavlovianinstrumental transfer procedure (nosepoke responses during the CS+ and CS -), upon which MK-801 administration did result in a reactivation-dependent impairment, the interpretation of this effect is complicated by the lack of discriminated approach shown by control saline-injected rats in the nonreactivated condition. As the analysis of the reactivated condition alone only revealed a trend toward an MK-801-induced impairment in goal-
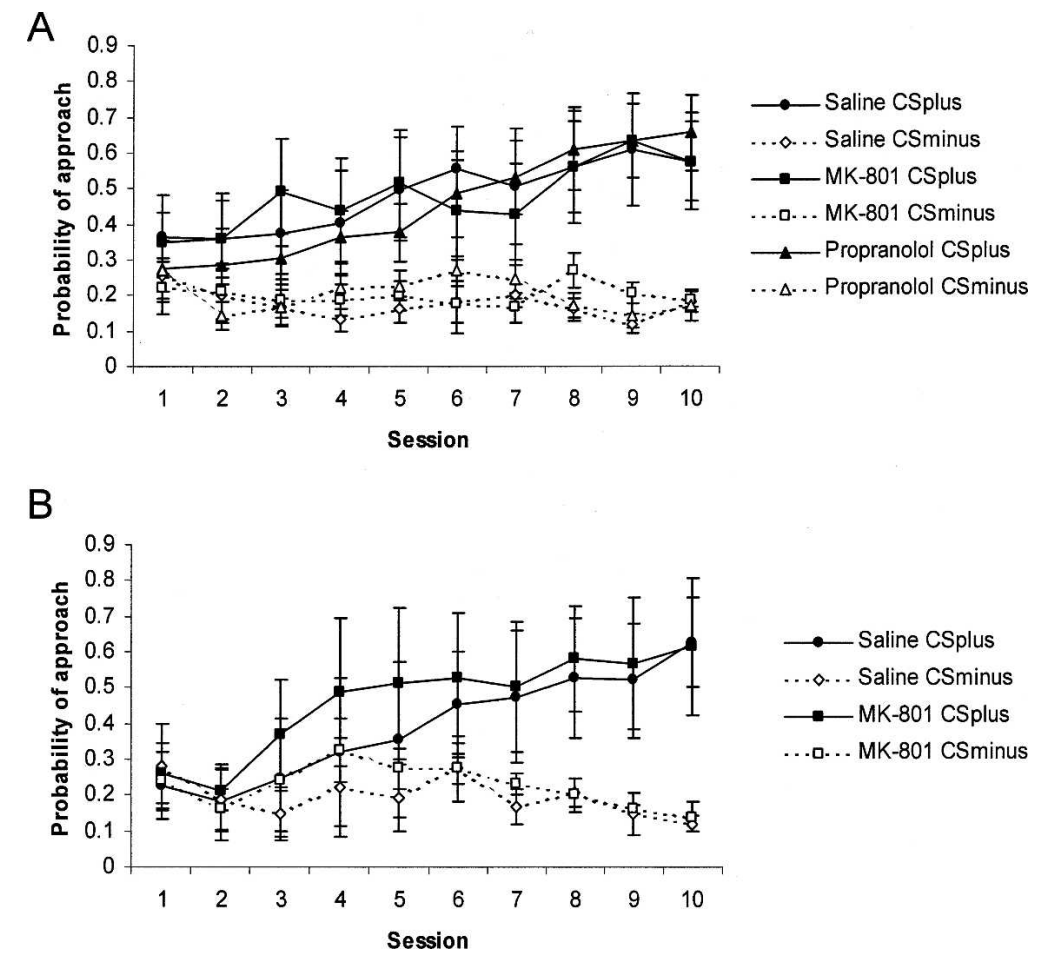

Figure 4. Acquisition of autoshaping. The probability of approaching the CS+ and CS - was measured over the $10 \mathrm{~d}$ of training for rats subsequently treated prior to memory reactivation $(A ; n=7$ for each group), and rats subjected to the nonreactivation control condition ( $B ; n=6$ for each group). There were no differences between the groups. Data presented as mean \pm SEM. 
A

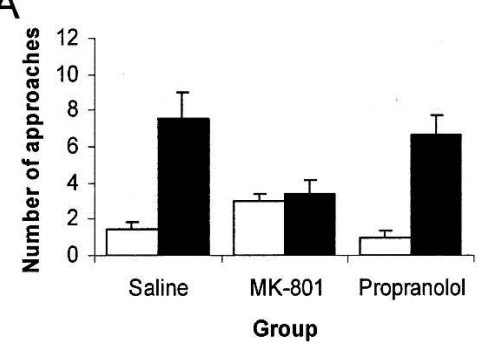

B

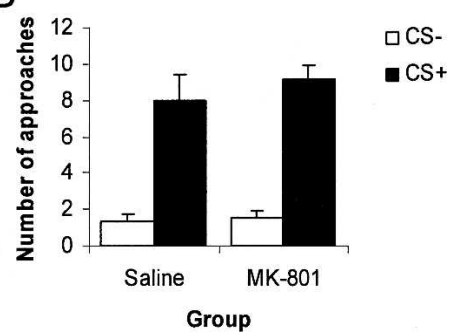

Figure 5. MK-801, but not propranolol, impaired subsequent autoshaping. The number of approaches to the CS+ and CS - were measured during a probe test. $(A)$ While rats administered saline or propranolol prior to memory reactivation showed greater approaches to the CS+ than the CS - , the administration of MK-801 eliminated such selective approach ( $n=7$ for each group). ( $B$ ) The effects of MK-801 were critically dependent upon memory reactivation, as omission of the reactivation session resulted in MK-801 having no effect on subsequent discriminative approach ( $n=6$ for each group). Data presented as mean + SEM.

tracking, it remains unclear whether the memory representations mediating Pavlovian conditioned goal-tracking undergo reconsolidation following their retrieval.

The present measures of general motivational Pavlovian properties are psychologically and neurally distinct from the conditioned reinforcing properties of appetitive stimuli that we have previously studied (Lee et al. 2005; Lee and Everitt 2008; Milton et al. 2008). While there is a common requirement for the nucleus accumbens core in conditioned reinforcement, Pavlovian-instrumental transfer and autoshaping (Parkinson et al. 1999, 2000b; Hall et al. 2001), there is a differential involvement of amygdala subnuclei. Whereas conditioned reinforcement depends critically upon the integrity of the basolateral amygdala (BLA) (Burns et al. 1993), but not the central amygdala (CeN) (Robledo et al. 1996), both Pavlovian-instrumental transfer and autoshaping depend instead upon the central, but not the basolateral, amygdala (Parkinson et al. 2000a; Hall et al. 2001). Therefore the effect of MK-801 on both autoshaping and Pavlovianinstrumental transfer observed here may reflect either their common neural substrates, or a similar dependence upon NMDA receptor-mediated neurotransmission in overlapping but distinct neural circuits.

In contrast to the selective effect of MK-801 upon the reconsolidation of the appetitive memory representations mediating Pavlovian-instrumental transfer and autoshaping reported here, we have previously demonstrated that both NMDA and $\beta$-adrenergic receptor antagonism, using the same drugs and doses, impair appetitive memory reconsolidation in a procedure that isolates and measures conditioned reinforcement (Lee and Everitt 2008; Milton et al. 2008). Therefore, the vulnerability of the general motivational tasks used here to propranolol is lower than other spatial, aversive, and sensory-specific appetitive memory representations (Przybyslawski et al. 1999; Debiec and LeDoux 2004; Milton et al. 2008). The dissociable effects of $\beta$ adrenergic receptor antagonism upon appetitive memory reconsolidation in sensory-specific and general motivational settings may therefore be related to their specific neural substrates. Given that the BLA has been shown to be a primary central locus of effect of systemically administered NMDA and $\beta$-adrenergic receptor antagonists in memory reconsolidation studies (Debiec and LeDoux 2004; Milton et al. 2008), it is likely that the common effect of MK-801 and propranolol to impair memory reconsolidation in conditioned reinforcement procedures reflects their actions in the BLA. In contrast, the CeN has yet to be defined as a locus of action of systemically applied amnestic agents in memory reconsolidation settings. Therefore, it is possible that in the present study, the amnestic effects on conditioned motivational representations of MK-801, but not propranolol, reflect the

consolidation in the present tasks may limit its potential application to the treatment of compulsive reward seeking (Milton et al. 2008). Sign-tracking behavior is associated with the psychomotor sensitization aspects of drug addiction (Flagel et al. 2008), and both the approach to and the general motivational effects of reward-associated stimuli play important roles in their control over behavior. Therefore, in order to maximize beneficial effects, any potential reconsolidation-based therapeutic strategy for compulsive reward seeking should impair all appetitive Pavlovian memory representations. The present data suggest that while NMDA receptor antagonism is likely to fulfill this requirement, it remains unclear whether antagonism of $\beta$-adrenergic receptors is capable of disrupting the reconsolidation of the general motivational and incentive properties of appetitive stimuli, and this may limit its potential clinical therapeutic utility.

\section{Materials and Methods}

\section{Subjects}

The subjects were 107 experimentally naïve adult male Lister Hooded rats, weighing 250-300 g. They were housed in pairs, in holding rooms maintained at $21^{\circ} \mathrm{C}$ on a reversed-light cycle $(12$ h light: $12 \mathrm{~h}$ dark; lights on at 19:00). Food was restricted to 15 g/day and water was freely available throughout the experiment. All procedures were conducted in accordance with the United Kingdom 1986 Animals (Scientific Procedures) Act (Project License PPL 80/1767).

\section{Drug administration}

(+)-MK-801 hydrogen maleate and ( \pm )-propranolol hydrochloride (Sigma, Poole) were both dissolved in sterile saline for intraperitoneal (i.p.) injection $(1 \mathrm{~mL} / \mathrm{kg})$. The doses of MK-801 $(0.1$ $\mathrm{mg} / \mathrm{kg}$ ) and propranolol (10 $\mathrm{mg} / \mathrm{kg}$ ) selected have previously been shown to impair the reconsolidation of both aversive and appetitive Pavlovian memories (Debiec and LeDoux 2004; Lee et al. 2006b; Lee and Everitt 2008; Milton et al. 2008).

\section{Behavioral apparatus}

All behavioral training and testing took place in 12 operant chambers (Med Associates), each measuring $29.5 \times 32.5 \times 23.5$ $\mathrm{cm}$. Three sides were constructed of steel and both the ceiling and front wall were perspex, the latter also serving as a door. On the front wall were located two modular low profile retractable levers. These were $4 \mathrm{~cm}$ wide, separated by $12 \mathrm{~cm}$, and raised $8 \mathrm{~cm}$ above the grid floor. Located above each lever was a white cue light, and between the levers was positioned a liquid dipper, which could be raised in order to present $0.1 \mathrm{~mL}$ of liquid reward into a reward magazine; a nosepoke entry into the magazine was detected by an infrared beam break. On the opposite, rear wall were located a white house light, a drop dispenser with liquid 
receptacle, and two auditory stimulus generators; a sonalert 2900 $\mathrm{Hz}$ tone generator, and a mechanical clicker module. Below the grid floor was a removable tray lined with absorbent paper.

\section{Behavioral procedures}

\section{Experiment 1: Pavlovian-instrumental transfer}

\section{Instrumental training}

The rats were placed individually in the operant chambers, into which a single lever was inserted at the beginning of the 30-min session, a response upon which was reinforced by a 5-sec elevation of the liquid dipper ( $0.1 \mathrm{~mL}$ of $20 \%$ sucrose). Instrumental training began under a random interval (RI) 2-sec schedule of reinforcement, and progressed through $9 \mathrm{~d}$ of training in the following sequence: RI2, RI2, RI2, RI15, RI30, RI60, RI60, RI60, RI60.

\section{Pavlovian conditioning}

Following acquisition of instrumental responding, the rats were conditioned to associate an auditory stimulus (CS+; $2.9 \mathrm{kHz}$ tone or $10 \mathrm{~Hz}$ clicker, counterbalanced) with the delivery of sucrose reward via the liquid dipper. In 24-min sessions, the CS+ was presented six times for 2 min with an interstimulus interval (ISI) of $2 \mathrm{~min}$, during which the liquid dipper was elevated on an RT30-sec schedule. The lever was not present at any time. Nosepoke responses into the reward magazine were measured during the CS and ISI in order to assess the acquisition of Pavlovian conditioning. On the tenth and final day of Pavlovian conditioning, a neutral unreinforced CS $-(10 \mathrm{~Hz}$ clicker or $2.9 \mathrm{kHz}$ tone, counterbalanced) was presented twice following the penultimate and ultimate presentations of the $\mathrm{CS}_{+}$, in a 32-min session. Furthermore, on the final $2 \mathrm{~d}$ of training, rats were also habituated to the intra-peritoneal injection procedure using the saline vehicle.

\section{Instrumental reminder, memory reactivation, and instrumental} extinction

Following Pavlovian conditioning, the rats were exposed first to a single instrumental reminder session (identical to instrumental training), and on the next day to a 24-min memory reactivation session that consisted of $6 \times 2$-min re-exposures to the CS+ with a 2-min ISI. This was essentially a Pavlovian extinction session, identical in all regards to the first nine sessions of Pavlovian conditioning except that the sucrose reward was unavailable. Rats were injected with saline, MK-801, or propranolol i.p. 30 min before the start of the memory reactivation session. Rats in the nonreactivated condition received injections in the holding room and were immediately returned to their home cages. Finally, prior to testing for Pavlovian-instrumental transfer, the rats received a single 30 -min instrumental extinction session, in which the lever was extended into the chamber, but responses were not reinforced. The purpose of the extinction session was to reduce baseline lever pressing rates to a level that enhances the sensitivity of responding to CS+ presentation (Dickinson et al. 2000; Hall et al. 2001).

\section{Pavlovian-instrumental transfer test}

The capacity of the Pavlovian CS+ to enhance instrumental responding was tested in a single 32 -min session. The lever was extended into the chamber and responses were measured during the 2-min ISIs and 2-min presentations of the CS+ and CS-. Each stimulus was presented four times through four cycles of the following sequence: ISI, clicker, ISI, tone. The data are presented as the ratio of responses during stimulus presentation relative to the previous ISI using the following formula: CS/(CS+ISI). Thus, a ratio of 0.5 represents no impact of CS presentation, and values greater than 0.5 reflect a transfer effect. As the potentiation of ongoing instrumental responding requires a baseline level of lever pressing, a criterion of greater than two responses in the sum of the final two ISIs was used. As instrumental responding extinguished significantly through the course of the transfer test, this criterion resulted in the omission of 22 out of the initial 56 subjects from the statistical analysis.

\section{Experiment 2: Autoshaping}

Training

Separate groups of rats were first subjected to two reinforcer familiarization sessions, in which the liquid sucrose reward was delivered on a VI 20-60 sec schedule. They were then trained over 10 sessions in a Pavlovian autoshaping procedure in the same chambers. Each session consisted of 50 trials, with a variable ITI between $10 \mathrm{sec}$ and $40 \mathrm{sec}$. During 25 trials, one lever was extended into the chamber and the light above it illuminated, both for $10 \mathrm{sec}$ (designated CS+; left or right, counterbalanced), immediately following which a $0.3-\mathrm{mL}$ drop of $20 \%$ sucrose was dispensed into the liquid receptacle on the opposite wall. The remaining 25 trials consisted of the presentation of the other lever and light (CS - ) for $10 \mathrm{sec}$, following which no reward was delivered. The order of presentation of the CS+ and CS - was random within each pair of presentations; hence the maximum number of consecutive presentations of each CS was two. An approach to the CS+ or CS - was measured by contact with the lever during its presentation, though importantly these contacts had no impact upon the delivery of the reinforcer (i.e., the sucrose was delivered noncontingently). The number of approaches to the CS+ and CS - out of the maximum 25 was recorded to give a probability of approach to each CS. On the final $2 \mathrm{~d}$ of training, rats were also habituated to the intra-peritoneal injection procedure using the saline vehicle.

\section{Memory reactivation}

On the third day following training, the rats were injected with saline, MK-801, or propranolol i.p. and $30 \mathrm{~min}$ later were returned to the chambers for a memory reactivation session. This session was identical to a training session, but no sucrose reward was available following either CS+ or CS - presentation. Rats in the nonreactivated condition received injections in the holding room and were immediately returned to their home cages.

Probe test

Three days later, the rats were given a probe test, which involved 20 simultaneous presentations of both the CS+ and CS - with no sucrose reward, and the first CS contact during the $10 \mathrm{sec}$ of CS presentation was recorded as a measure of discriminated approach.

\section{Statistical analysis}

Data are presented as mean + SEM lever presses, ratio of responses or approaches. The data were checked for sphericity, and the Greenhouse-Geisser correction was used as appropriate prior to ANOVA. A significance level of $P<0.05$ was selected for all analyses.

\section{Acknowledgments}

This work was supported by a grant from the UK Medical Research Council (program grant no. 9536855) and was conducted within the MRC/Wellcome Trust Behavioural and Clinical Neuroscience Institute.

\section{References}

Blaiss, C.A. and Janak, P.H. 2007. Post-training, but not post-reactivation, administration of amphetamine and anisomycin modulates Pavlovian conditioned approach. Neurobiol. Learn. Mem. 87: 644-658.

Burns, L.H., Robbins, T.W., and Everitt, B.J. 1993. Differential-effects of excitotoxic lesions of the basolateral amygdala, ventral subiculum and medial prefrontal cortex on responding with conditioned reinforcement and locomotor-activity potentiated by intraaccumbens infusions of D-amphetamine. Behav. Neurosci. 55: $167-183$.

Cador, M., Robbins, T.W., and Everitt, B.J. 1989. Involvement of the amygdala in stimulus reward associations-Interaction with the ventral striatum. Neuroscience 30: 77-86. 
Cardinal, R.N., Parkinson, J.A., Hall, J., and Everitt, B.J. 2002. Emotion and motivation: The role of the amygdala, ventral striatum, and prefrontal cortex. Neurosci. Biobehav. Rev. 26: 321-352.

Corbit, L.H. and Balleine, B.W. 2005. Double dissociation of basolateral and central amygdala lesions on the general and outcome-specific forms of Pavlovian-instrumental transfer. J. Neurosci. 25: 962-970.

Debiec, J. and LeDoux, J.E. 2004. Disruption of reconsolidation but not consolidation of auditory fear conditioning by noradrenergic blockade in the amygdala. Neuroscience 129: 267-272.

Di Ciano, P., Cardinal, R.N., Cowell, R.A., Little, S.J., and Everitt, B.J. 2001. Differential involvement of NMDA, AMPA/kainate, and dopamine receptors in the nucleus accumbens core in the acquisition and performance of Pavlovian approach behavior. $J$. Neurosci. 21: 9471-9477.

Dickinson, A., Smith, J., and Mirenowicz, J. 2000. Dissociation of Pavlovian and instrumental incentive learning under dopamine antagonists. Behav. Neurosci. 114: 468-483.

Diergaarde, L., Schoffelmeer, A.N., and De Vries, T.J. 2006. Beta-adrenoceptor mediated inhibition of long-term reward-related memory reconsolidation. Behav. Brain Res. 170: 333-336.

Eisenberg, M., Kobilo, T., Berman, D.E., and Dudai, Y. 2003. Stability of retrieved memory: Inverse correlation with trace dominance. Science 301: 1102-1104.

Estes, W.K. 1943. Discriminative conditioning. I. A discriminative property of conditioned anticipation. J. Exp. Psych. 32: 150-155.

Everitt, B.J., Cardinal, R.N., Hall, J., Parkinson, J.A., and Robbins, T.W. 2000. Differential involvement of amygdala subsystems in appetitive conditioning and drug addiction. In The amygdala: A functional analysis, (ed. J.P. Aggleton), pp. 353-390. Oxford University Press, Oxford, UK.

Flagel, S.B., Watson, S.J., Akil, H., and Robinson, T.E. 2008. Individual differences in the attribution of incentive salience to a reward-related cue: Influence on cocaine sensitization. Behav. Brain Res. 186: 48-56.

Hall, J., Parkinson, J.A., Connor, T.M., Dickinson, A., and Everitt, B.J. 2001. Involvement of the central nucleus of the amygdala and nucleus accumbens core in mediating Pavlovian influences on instrumental behaviour. Eur. J. Neurosci. 13: 1984-1992.

Konorski, J. 1967. Integrative activity of the brain. Chicago University Press, Chicago.

Lee, J.L.C. and Everitt, B.J. 2008. Appetitive memory reconsolidation depends upon NMDA receptor-mediated neurotransmission. Neurobiol. Learn Mem. 90: 147-154.

Lee, J.L.C., Di Ciano, P., Thomas, K.L., and Everitt, B.J. 2005. Disrupting reconsolidation of drug memories reduces cocaine seeking behavior. Neuron 47: 795-801.

Lee, J.L.C., Milton, A.L., and Everitt, B.J. 2006a. Cue-induced cocaine seeking and relapse are reduced by disruption of drug memory reconsolidation. J. Neurosci. 26: 5881-5887.
Lee, J.L.C., Milton, A.L., and Everitt, B.J. 2006b. Reconsolidation and extinction of conditioned fear: Inhibition and potentiation. $J$. Neurosci. 26: 10051-10056.

Lewis, D.J. 1979. Psychobiology of active and inactive memory. Psychol. Bull. 86: 1054-1083.

Lovibond, P.F. 1983. Facilitation of instrumental behavior by a Pavlovian appetitive conditioned stimulus. J. Exp. Psychol. Anim. Behav. Process. 9: 225-247.

Milekic, M.H., Brown, S.D., Castellini, C., and Alberini, C.M. 2006. Persistent disruption of an established morphine conditioned place preference. J. Neurosci. 26: 3010-3020.

Miller, C.A. and Marshall, J.F. 2005. Molecular substrates for retrieval and reconsolidation of cocaine-associated contextual memory. Neuron 47: 873-884.

Milton, A.L., Lee, J.L.C., and Everitt, B.J. 2008. Reconsolidation of appetitive memories for both natural and drug reinforcement is dependent on $\beta$-adrenergic receptors. Learn. Mem. 15: 88-92.

Nader, K. 2003. Memory traces unbound. Trends Neurosci. 26: 65-72.

Nader, K., Schafe, G.E., and Le Doux, J.E. 2000. Fear memories require protein synthesis in the amygdala for reconsolidation after retrieval. Nature 406: $722-726$.

Parkinson, J.A., Olmstead, M.C., Burns, L.H., Robbins, T.W., and Everitt, B.J. 1999. Dissociation in effects of lesions of the nucleus accumbens core and shell on appetitive Pavlovian approach behavior and the potentiation of conditioned reinforcement and locomotor activity by D-amphetamine. J. Neurosci. 19: 2401-2411.

Parkinson, J.A., Robbins, T.W., and Everitt, B.J. 2000a. Dissociable roles of the central and basolateral amygdala in appetitive emotional learning. Eur. J. Neurosci. 12: 403-413.

Parkinson, J.A., Willoughby, P.J., Robbins, T.W., and Everitt, B.J. 2000b. Disconnection of the anterior cingulate cortex and nucleus accumbens core impairs Pavlovian approach behavior: Further evidence for limbic cortical-ventral striatopallidal systems. Behav. Neurosci. 114: 42-63.

Przybyslawski, J., Roullet, P., and Sara, S.J. 1999. Attenuation of emotional and nonemotional memories after their reactivation: Role of beta adrenergic receptors. J. Neurosci. 19: 6623-6628.

Robledo, P., Robbins, T.W., and Everitt, B.J. 1996. Effects of excitotoxic lesions of the central amygdaloid nucleus on the potentiation of reward-related stimuli by intra-accumbens amphetamine. Behav. Neurosci. 110: 981-990.

Valjent, E., Corbille, A.G., Bertran-Gonzalez, J., Herve, D., and Girault, J.A. 2006. Inhibition of ERK pathway or protein synthesis during reexposure to drugs of abuse erases previously learned place preference. Proc. Natl. Acad. Sci. 103: 2932-2937.

Received April 14, 2008; accepted in revised form June 11, 2008. 


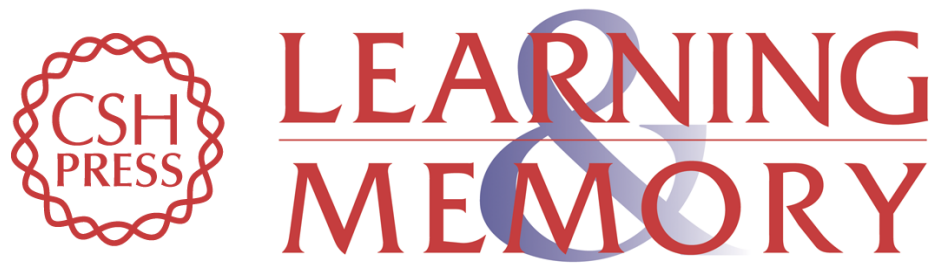

\section{Reactivation-dependent amnesia in Pavlovian approach and instrumental transfer}

Jonathan L.C. Lee and Barry J. Everitt

Learn. Mem. 2008, 15:

Access the most recent version at doi:10.1101//m.1029808

References This article cites 30 articles, 10 of which can be accessed free at:

http://learnmem.cshlp.org/content/15/8/597.full.html\#ref-list-1

License

Email Alerting Receive free email alerts when new articles cite this article - sign up in the box at the Service top right corner of the article or click here. 